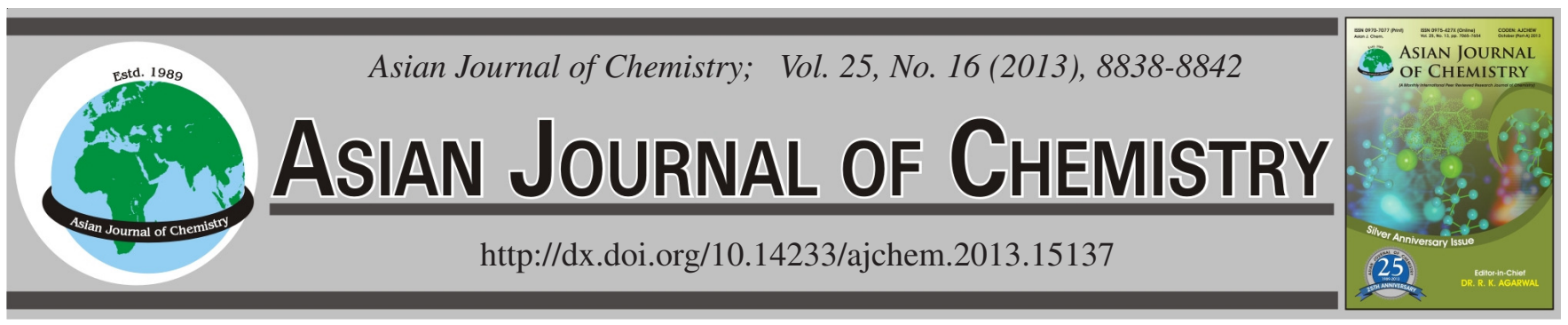

REVIEW

\title{
Applications of Microcalorimetry in Environmental Sciences
}

\author{
X.Q. Ning, W.W. QiaO, L. Zhang and X. GAO*
}

Eco-Environment in Three Gorges Reservoir Region, Ministry of Education, Chongqing University, Chongqing 400045, P.R. China

*Corresponding author: E-mail: gaoxu@cqu.edu.cn

(Received: 14 January 2013;

Accepted: 2 September 2013)

AJC-14063

Techniques of microcalorimetry have been proved a powerful tool to elucidate thermodynamic information on various reactions and processes during the past two decades. In addition to their application in fundamental research, the studies on the environmental sciences have been established in some areas. This review mainly focuses on the use of microcalorimetry in the determination of microbial activity in soil and the wastewater treatment processes. Promising prospects for the applications of microcalorimetry in environmental sciences are also discussed.

Key Words: Environmental, Isothermal microcalorimetry, Microbial activity, Microcalorimeter.

\section{INTRODUCTION}

In nature, all the physical, chemical and biological processes are accompanied by heat effects, which make calorimetry useful as a general technique for thermodynamic and analytical investigations without any interference in these processes. The non-specific calorimetric signal from a complex system is usually difficult to interpret on a molecular level, in the absence of more specific analytical information. An important technique is the isothermal microcalorimetry, which has received considerable attention in the broad field of modern science and technology. The term 'microcalorimeter' usually implies a sensitive calorimeter that is useful for measurements in the microwatt range, under essentially conditions. In the microcalorimetric study, microcalorimeter is used in thermodynamic work, in kinetics and as analytical tools to obtain the both reaction kinetics and thermodynamic parameters such as microbial growth rate constant $\mathrm{k}$, the heat yield of the microbial growth $\mathrm{Q}_{\mathrm{T}}$, the metabolic enthalpy $\Delta \mathrm{H}_{\text {met }}$ etc. ${ }^{1,2}$. It can provide qualitative and a real-time quantitative data that has proven to be indispensable for a wide variety of applications: from determining stability of inanimate materials to the overall metabolism of microorganism, cell and tissue cultures as well as more complex biological system, under 'normal' and modified environmental condition ${ }^{3,4}$. Besides, an advantage of the microcalorimetric method is its simplicity and non-disturbance of samples. Signals could be continuously recorded for the same sample for a prolonged period of time, which is not possible using other methods ${ }^{5}$. Actually, the microcalorimetric method has been employed over a wide range of studies involving life science, material science and pharmaceutical development over the past years ${ }^{6-8}$.

In the environmental system, various complex physical, chemical and biochemical reactions take place all times and these reactions are associated with more or less heat release which can be conveniently monitored by microcalorimetry. Due to environmental system always is a complex ecosystem; microcalorimetry is attractive because of its particularly well suited for the discovery and quantitative assessment of unexpected or unknown phenomena. The aim of the present review is to show how the microcalorimetric method is applied in environmental sciences, what kind of information it can provide and what the perspectives of further developments of this method are.

Microcalorimeters: The instruments used in microcalorimetry mainly fall into two categories: heat conduction and adiabatic and power compensation. The application of microcalorimetry to environment science is focus on the biological processes. Due to all the biological processes are almost under isothermal condition, isothermal microcalorimeter is usually used in the environmental science. Most isothermal microcalorimeters currently used in measuring of living systems are of the heat conduction type ${ }^{1}$. In heat conduction microcalorimeter, the heat released (or absorbed) in the reaction vessel is allowed to flow to a surrounding heat sink, normally a metal block surrounding the vessel. A thermopile which was positioned between the reaction vessel and the heat sink is used as a sensor to record the heat flow ${ }^{9}$. And almost all isothermal microcalorimeters are designed as twin instruments. 
The LKB 2277 Bioactivity Monitor which is a type of heat conduction microcalorimeter is designed to monitor continuously a wide variety of processes and complex systems over the temperature range $20-80^{\circ} \mathrm{C}$. Each measuring cylinder normally contains a sample and a reference in separate measuring cups (twin system). The detection limit of this system is $0.15 \mathrm{~mW}$ and the baseline stability (over a period of $24 \mathrm{~h}$ ) is $\pm 0.2 \mathrm{~mW}$. The temperature control in the LKB 2277 Bioactivity Monitor is constant temperature water bath system ${ }^{10,11}$. For fundamental research and method developments of low sample throughputs, a twin microcalorimeter can be accepted. However, for the process monitoring which normally requires a high sample throughput, so "multi-channel" instruments is more appropriate. In a multi-channel heat conduction microcalorimeter several microcalorimeter normally share the same heat sink. One (or more) of the microcalorimeters is used as reference(s) for the other units, or the multi-channel instrument is made up by several twin microcalorimeters ${ }^{1}$.

The TAM III multi-channel thermal activity monitor is a "48 channel" isothermal microcalorimeter which is designed to monitor a wide variety of processes and complex systems continuously such as the thermal activity of physical, chemical and biological processes in terms of heat, heat flow and heat capacity over a temperature range of $15-150^{\circ} \mathrm{C}$. In this instrument each channel is a twin microcalorimeter where the two units are positioned with one on the top and the other at the bottom. The 48 tube-shaped twin microcalorimeters are all inserted into a precise liquid thermostate (oil, $15-150^{\circ} \mathrm{C}$, short term noise within $0.1 \mathrm{~mW}$, baseline drift during $24 \mathrm{~h}$ within $0.2 \mathrm{~mW}$ ). One of the TAM III multi-channels microcalorimetric units is used as a reference. All vessels are simultaneously introduced into the sample chamber ${ }^{12,13}$.

Compared to other calorimeters, the microcalorimeter named Bio-RC1 is suitable for biological studies in the liquid form since the vessel has a design comparable to a standard lab-scale bio-reactor in which the content can be agitated, aerated and $\mathrm{pH}$ can be controlled, under stable isothermal conditions. Furthermore, a great advantage of this microcalorimeter is that samples can be extracted from the medium where calorimetric measurements are carried out, without disturbing the process and without giving any significant signal alteration $^{14,15}$. The system is also equipped with a dissolved oxygen probe and with a $\mathrm{pH}$ probe directly inserted into the reaction vessel. Heat production rate, dissolved oxygen (DO) concentration and $\mathrm{pH}$ are on-line acquired and processed by Labview (National Instruments). For investigate biological processes, the system has a resolution of 5-10 $\mathrm{mW} \mathrm{L}^{-1}$ and the stability of the reaction volume temperature to $\pm 1 \mathrm{mK}$ for $12 \mathrm{~h}^{16}$.

The Bio-RC1 can be operated in isothermal, isoperibolic (constant jacket temperature) or adiabatic conditions. In the isothermal mode, a low-viscosity silicone oil is pumped at a high rate $\left(2 \mathrm{Ls}^{-1}\right)$ through the reactor jacket in order to maintain a constant temperature of the reactant medium $\left(\mathrm{T}_{\mathrm{r}}\right)$ by proper control of the jacket temperature $\left(\mathrm{T}_{\mathrm{j}}\right)$. The jacket temperature is carefully controlled by blending oil from a 'hot' and a 'cold' oil circuit via an electronically controlled metering valve. Therefore, when a process dissipates or takes up heat, $T_{j}$ increases or decreases, respectively. The resulting gradient across the reactor wall is directly proportional to the thermal power liberated or absorbed by the process. The resulting temperature gradient across the reactor wall is directly proportional to the thermal power liberated or absorbed by the process $\left(\mathrm{Q}_{\mathrm{r}}\right)$ according to:

$$
\mathrm{Q}_{\mathrm{r}}=\mathrm{UA} \times\left(\mathrm{T}_{\mathrm{r}}-\mathrm{T}_{\mathrm{j}}\right)
$$

where $\mathrm{U}$ is the overall heat-transfer coefficient $\left(\mathrm{W} \mathrm{m}^{-2} \mathrm{~K}^{-1}\right), \mathrm{A}$ is the heat transfer surface $\left(\mathrm{m}^{2}\right)$ and $\left(\mathrm{T}_{\mathrm{r}}-\mathrm{T}_{\mathrm{j}}\right)$ the temperature difference $(\mathrm{K})$ between the reactor contents and the jacket oil. When a new sample is put into the calorimeter, the UA factor has to be experimentally determined using an internal electrical calibration heater of known power output ${ }^{17,18}$.

The Bio-RC1 does not require any energy balance to be computed and, therefore, it is possible to carefully monitor and control important process variables such as temperature and medium agitation and to operate the vessel under constant and reproducible process conditions. In fact, heat production rate due to biological exogenous reactions $\left(\mathrm{Q}_{\mathrm{ex}}\right)$ is simply equal to the measured heat flux $\left(\mathrm{Q}_{\mathrm{r}}\right)$ corrected for the baseline contribution, assumed to be constant throughout the test, which is the sum of two terms: the external heat loss and gain terms (e.g. due to stirring, evaporation and condensation effects, heat losses to the environment) and the thermal power dissipation related to microbial endogenous metabolism ${ }^{16}$.

Applications in determination of microbial activity: Microorganisms are essential to the environment due to their role in the interchange of energy and matter in the system. Microorganisms are responsible for various biochemical reactions such as in decomposing organic materials, cycling mineral compounds, promoting/suppressing plant growth. Microbial populations in the environment exhibit dynamics changes and their biodiversity and functions are influenced by various factors. Microbial ecology focuses on microbial diversity including the identification and quantification of microorganism and microbial activity, that is, what the microorganisms are doing in their habitats ${ }^{5}$. As a result of the microbial activity, the reactions and processes generate a flow of heat, which is easily detected by a microcalorimeter.

For instance, in the studies of the soil microbial activity, the correlation between the soil characteristics (such as soil moisture, organic matter content, microbial biomass and agricultural activities) and microbial activities can be demonstrated by the microcalorimetry. Barros et al. ${ }^{19}$ studied the effect of moisture on the population and activity of soil microbes by the microcalorimetric method and establish the relationships between heat evolution and the microbial activity of forest soil in Santiago, Spain. A positive correlation was found between the percentage humidity, the total heat evolution and the microbial growth rate constant. Prado et al. ${ }^{20}$ confirmed that moisture has an effect on microbial activity of soils. The microbial activity has a maximum value between 36.74 and $37.74 \%$ in real moisture values. Above $41.82 \%$ of real moisture, the total heat decreases. They suggested that the moisture applied to any experimental system is a decisive factor to optimize experiments in the microcalorimetry of soils. Critter et al. ${ }^{21}$ studied the microbial activity of isolates of bacteria and fungi of Brazilian soils amended with organic material (cattle manure, municipal refuse compost, earthworm casts and agrochemical triuralin) by the microcalorimetric method. The results illustrated that the organic material influenced the microbial activity and that bacteria and fungi activities were favoured on cattle manure. Barros ${ }^{22}$ also applied the microcalorimetric method to study the microbial activity of 
four soils sampled in different places of Galicia, a region in the Northwest of Spain. They found the changes in the soil microbial activity related to the percentage of organic matters. Their results showed that the soil with the highest percentage of organic matter generated the highest total heat production. Maximal microbial growth rate constant was found in the wineproducing land soil containing less organic matter, possibly because of a faster degradation of organic matter in this soil. Critter et al. ${ }^{23}$ measured microbial biomass C. with both oxygen-consumption and heat production. The results of both methods were, in general, highly correlated $(p<0.01)$. Significant correlations were also found between the results of both methods for measuring microbial activity. Critter et al. ${ }^{24}$ also investigated the correlation between the calorimetric data and the number of bacteria and fungi as a quantification of the microbial activity counted during 85 days. The results of simultaneous application of the two methods showed the correlation with $\mathrm{r}=0.8181$ and $p=0.0131$ for bacteria and $\mathrm{r}=$ 0.8134 and $p=0.014$ for fungi, over the period studied. They also compared in a same system, the thermal effect assessed through microcalorimetry with a classical microbiological method, the microbial carbon determination. Both methods showed a coefficient of correlation $r=0.7443$ and the statistical probability of occurrence of the event, $p<0.0001$. And the thermal effect could reflect the interactive effect in the soil microenvironment and other characteristics of the process, like enzymatic reactions and thermal degradation of compounds available for growth. Zheng et al. ${ }^{5}$ investigated the influence of different agricultural practices and vegetations on soil microbial activity by microcalorimetric technique. The soil samples were divided into two groups differing in power-time curves. Regueira $e t a l .{ }^{25}$ also found that the microbial growth rate constant was higher in maize field than that in uncultivated Unibric Regosol using microcalorimetry.

To the biological wastewater treatment processes, few works are found in literature about the applicability of microcalorimetry. However, because of the universality of enthalpy changes in biological reactions, calorimetric techniques can also be used in anoxic and anaerobic condition of wastewater treatment processes. Beaubien et al. ${ }^{26}$ applied the flow microcalorimetric method to study the microbial activity of activated sludge (aerobic) treatment of industrial wastewater. Comparison between oxygen consumption and the microcalorimetric method was also studied, a linear correlation was obtained between the two parameters $(r=0.97)$. Jolicoeur et $a l .{ }^{27}$ also monitored the biological activity of two industrial wastewater treatment processes: an activated sludge (aerobic) treatment of a textile factory effluent and a methane fermentation (anaerobic) treatment of effluents from a cheese factory with flow microcalorimeter. In both types of system, the heat flux resulting from metabolic reactions during bacterial degradation of organic substances in the effluent could be measured adequately. Aulenta et al. ${ }^{14}$ pointed out the excellent agreement between microcalorimetric and respirometric data, obtained simultaneously under aerobic conditions using activated sludge from a lab-scale scale reactor. And a possible combination of the two types of measurements could also make it possible to identify the presence of particular metabolisms (e.g. unbalance of growth conditions, futile cycles and simultaneous aerobic and anaerobic metabolisms). Microcalorimetry allows a direct comparison between aerobic and anoxic bioprocesses by using the same experimental (respirometric) approach and instrument.

Besides, the use of a high-resolution calorimeter made possible to measure even relatively weak exothermic biological processes like autotrophic nitrification. Daverio et al. ${ }^{18}$ presented the biokinetic characterization of nitrifying population in activated sludge with the application of calorimetric measurements. Thermograms related to biological nitrification reactions (ammonia or nitrite consumption) was interpreted and heat yields related to ammonia and nitrite consumption was evaluated. And the correlation between calorimetric data and oxygen uptake has been verified and oxycaloric equivalents have been estimated for both ammonia oxidizers ( $\mathrm{r}=$ $0.978)$ and nitrite oxidizers $(\mathrm{r}=0.996)$. Menert et $a l .^{28}$ measured the growth rates of sulphate-reducing bacteria (SRB) isolated from anaerobic sludge of yeast factory wastewater treatment plant. The maximum growth rates $\mu_{\max }$ for SRB determined by microcalorimetry and ATP analysis were different $-\mu_{\max }(\mathrm{dQ} / \mathrm{dt})=0.165 \pm 0.008 \mathrm{~h}^{-1}$ and $\mu_{\max }\left(\mathrm{N}_{\mathrm{ATP}}\right)=$ $0.207 \pm 0.013 \mathrm{~h}^{-1}$. They explained the reason may lie in greater proportion of maintenance energy at low growth yields in the lag phase and in the early exponential phase. In calculation of growth rates from power-time curves the main prerequisite is the constancy of the stoichiometry of biomass during the exponential growth.

Applications in assessment of microbial activity in polluted environment: During the last 50 years, many anthropogenic xenobiotics have been extensively used to ensure the human healthy or harvest of agricultural products. But most of anthropogenic xenobiotics application, however, never reaches its target organism is finally excess introduced into soil and water. Microorganisms are an important biological component and play vital roles in environmental ecosystem structure and stability through their roles in nutrient cycling and organic matter decomposition. Thus, it is necessary to improve the ability for predicting the consequences of anthropogenic xenobiotics and then implement corrective actions in a short time due to their possible toxic effects on the environment and threats to human health.

Microcalorimetry can also be applied in studies on microbial activity in polluted environment such as pesticides, toxic agents and heavy metal. Prado et al. $^{29}$ applied microcalorimetry in a series of experiments to follow the effect caused by the pesticide 2,4-dichlorophenoxyacetic acid $(2,4-$ D) on microbial activity of red Latosol soil. They observed power-time curves recorded on calorimeter were followed by the increasing amount of the pesticide 2,4-D, from 0 to $10 \mathrm{mg}$, which affected directly the total thermal effect evolved by microorganisms. The curves showed a synergism on total thermal effect obtained by addition of $1 \mathrm{mg}$ of pesticide, causing a consumption of 2,4-D by microorganisms, as a new source of nutrients. However, above this mass, the total thermal effect decreased exponentially with increase of the amount of pesticide. These findings suggested heavy loads of 2, 4-D affected the life in this ecosystem due to the increase of the toxic products formed from degraded 2,4-D. Their finding also revealed that there was a synergistic effect in the degradation of 2,4-D and glucose by microorganisms. The influence of diuron $(3-(3,4-$ dichlorophenyl)-1,1-dimethylurea) and picloram (4-amino3,5,6-trichloro-picolinic acid) on microbial activity was also investigated by Prado and Airoldi ${ }^{30}$. Both free and immobilized 
pesticides were applied on typical Brazilian agricultural soil and the toxic effects on microbial activity were measured by microcalorimetry. The increasing amounts of diuron and picloram, either free or immobilized, caused a decrease of the original thermal effect. The diuron application of $333.33 \mu \mathrm{g} / \mathrm{g}$ causes a decrease of $96.4 \%$ of total microbial activity, while for anchored onto silica gel this dose causes a decrease of only $39.5 \%$. The same results was found soil microbial activity decreased by $87.4 \%$ in the presence of $10.00 \mu \mathrm{g} / \mathrm{g}$ free picloram, whereas only a decrease by $54.6 \%$ was detected for the same amount of immobilized picloram anchored onto silica gel. The calorimetric data showed that the anchored pesticide presented a much lower toxic effect than free diuron and picloram on microbial activity. Wang et al. ${ }^{31}$ evaluated the inhibitory effects of the pesticide Chlorpyrifos (CPF) and its oxon derivative (CPO) on soil microbial activity through the measurement of metabolic parameters. It was concluded that the toxic effect of CPF and CPO enhanced with increasing their doses and those microbial activities were promoted at lower doses to some extent. In addition, the acute toxicity of CPO $(0.37 \mu \mathrm{g} / \mathrm{g})$ was 26 times that of CPF $(9.8 \mu \mathrm{g} / \mathrm{g})$ by comparing the $20 \%$ inhibitory ratio $\mathrm{IC}_{20}$, illuminating reactive oxon derivative of chlorpyrifos was significantly more toxic than parental form. Zhuang et al. ${ }^{32}$ evaluated the effect of $\beta$-CYP on soil microbial by isothermal microcalorimetry. Their work revealed the very small impact of $\beta$-CYP at high concentrations in soil. Furthermore, the thermokinetic parameters obtained by microcalorimetry are in good agreement with the activities of the soil enzymes by enzyme assay. Chen et al. ${ }^{33}$ analyzed the toxic effects of diphenol species (catechol, resorcinol and hydroquinone) on soil microbial activity to a set of soil microorganisms with microcalorimetric method. The toxicity of the three diphenols was found in a descending sequence i.e., hydroquinone, resorcinol and catechol. The thermokinetic parameters obtained by microcalorimetry were in good agreement with the number of cultivable microorganisms, WSC and enzymatic activities. Guo et al. ${ }^{34}$ investigated the influence of petroleum contamination on soil microbial activities of 13 soil samples by means of calorimetry. Their study evaluated the effect of crude oil contamination on total soil metabolism from curves of metabolic heat rate and $\mathrm{Q}_{\text {total }}$. They found microcalorimetric metabolic analysis together with measurements of microbial number and urease activity is a useful method for evaluation of soil response to petroleum contamination. Concentrations of toxic metals in the environment are also a major ecological and toxicological concern due to their effects on the environment and threats to human health. On the basis of microcalorimetry method, McGuinness et al. ${ }^{35}$ investigated acute toxicity of heavy metals including $\mathrm{Hg}, \mathrm{Cd}, \mathrm{Cu}, \mathrm{Pb}, \mathrm{Cr}$, $\mathrm{V}$ and As. Because heat production was directly related to overall metabolism, inhibition of cell metabolic processes by toxicant reflected by heat output was better than chemiluminescence. Wang et al. ${ }^{36}$ studied toxic effect caused by the trivalent iron on the single and mixed microbes in sterilized soil that was inoculated with the single Bacillus subtilis (B. subtilis) (prokaryotic bacterium), single Candida humicola (C. humicola) (eukaryotic fungus) and the mixed-species by microcalorimetric technique. The results showed that the mixed-species have moderate tolerance to the iron overload, comparing with single species and exhibit synergistic interaction in exponential growth phase $(0-400.0 \mu \mathrm{g} / \mathrm{L})$. Meanwhile, there is no much difference in the thermal effect (Q) per gram soil sample for the single and mixed culture. Yao et al. ${ }^{13}$ studied soil microbial activity in the presence of different concentrations of hexavalent chromium $\left(\mathrm{K}_{2} \mathrm{Cr}_{2} \mathrm{O}_{7}\right)$ with a multi-channel thermal activity monitor. The results show that the poisonous species of $\mathrm{K}_{2} \mathrm{Cr}_{2} \mathrm{O}_{7}$ at a half inhibitory concentration $\left(\mathrm{IC}_{50}\right)$ value of 4:27 $\mu \mathrm{g} / \mathrm{L}$ against soil microbe and an increase of the amount of hexavalent chromium is associated to a decrease in the microbial activity of the soil. They suggested that microcalorimetry is a fast, simple and more sensitive method that can be easily performed to study the toxicity of different species of heavy metals on microorganism compared to other biological methods. Zhou et al. ${ }^{37}$ analyzed the effects of different concentrations of $\mathrm{Zn}$ on soil microbial communities and activities by loading five different doses of $\mathrm{Zn}(160-6000 \mu \mathrm{g} /$ $\mathrm{g}$ ) into a wheat surface soil. The microbial metabolic process revealed a significant bimodal pattern at high concentrations of $\mathrm{Zn}(>1920 \mu \mathrm{g} / \mathrm{g})$. It is suggested that soil microorganisms were very sensitive to zinc poisoning. The thermokinetic parameters obtained by microcalorimetry are in good agreement with the number of cultivable microorganisms and other soil quality parameters. Wang et al. ${ }^{38}$ evaluated the toxic effect of heavy metals ( $\mathrm{As}, \mathrm{Pb}, \mathrm{Cr}, \mathrm{Cd}, \mathrm{Cu}, \mathrm{Zn}$ and $\mathrm{Co}$ ) on the soil microbial activities and community. The microbial growth rate constant $(\mathrm{k})$, total heat evolution $\left(\mathrm{Q}_{\mathrm{T}}\right)$, metabolic enthalpy $\left(\Delta \mathrm{H}_{\text {met }}\right)$ and mass specific heat rate $\left(\mathrm{J}_{\mathrm{Q} / \mathrm{S}}\right)$ and microbial biomass $\mathrm{C}$ of soil samples were analyzed. According to the values of $\Delta \mathrm{H}_{\text {met }}$, a general order of inhibition was found to be $\mathrm{Cr}>\mathrm{Pb}>$ As $>\mathrm{Co}>\mathrm{Zn}>\mathrm{Cd}>\mathrm{Cu}$. Among these metals, $\mathrm{Cr}$ exhibited the strongest toxicity to soil microbes.

To the wastewater treatment processes, Daverio et al. ${ }^{39}$ investigated the response of an anaerobic granular sludge to increasing formaldehyde concentrations. They found that formaldehyde could be readily degraded and the toxicant dose led to $27 \%$ inhibition on glucose consumption; a $63 \%$ inhibition was observed when formaldehyde concentration was increased up to $3,600 \mathrm{mg} / \mathrm{L}$. No further biomass activity was detected after a $7200 \mathrm{mg} / \mathrm{L}$ formaldehyde pulse. Aulenta et al ${ }^{14}$ studied the toxic effect of $\mathrm{Hg}^{2+}$ on the activity of all microbial communities from activated sludge under aerobic and anoxic conditions. The toxicant led immediately to $100 \%$ inhibition of biological activity in the absence of $\mathrm{Hg}^{2+}(4.2 \mathrm{mg} / \mathrm{L})$ under aerobic conditions. In the nitrification aerobic batch tests, the addition of $\mathrm{Hg}^{2+}(0.8 \mathrm{mg} / \mathrm{L})$ led to $52 \%$ inhibition of nitrifying activity. But in the anoxic batch tests, the inhibition of $\mathrm{Hg}^{2+}$ increased from $6 \%$ (immediately after toxicant addition) to $23 \%$ after $2.5 \mathrm{~h}$ with a lower concentration $(1 \mathrm{mg} / \mathrm{L})$. They explained the retarded inhibition effect assuming that $\mathrm{Hg}^{2+}$ initially was adsorbed or slowly diffusing through the flocs and therefore only a small part of microorganisms forming the floc was affected by the toxicant; the delay in inhibition effect was then controlled by the diffusion of $\mathrm{Hg}^{2+}$ inside the floc. Chen et al..$^{40}$ evaluated the solvent tolerance of four strains of Pseudomonas sp. B1 and J2, Acinetobacter sp. B2 and J6 which were isolated from the activated sludge to benzene or toluene four strains by microcalorimetry. The microbial growths obtained by microcalorimetry were in good agreement with the results measuring the optical density of cultural medium at $600 \mathrm{~nm}$ (OD 600). They also examined the adverse effect of individual phenolic compounds (catechol, resorcinol and hydroquinone) and the interactive effect of the binary and 
tertiary mixtures on Bacillus subtilis (B. subtilis) which is widely distributed in soil and in putrefactive organisms using microcalorimetric method. Their results revealed that the toxicity of the investigated diphenols follows a descending sequence i.e., catechol, resorcinol, hydroquinone. Catechol and hydroquinone mixture exerted synergistic joint action while the other mixtures display additive joint actions. Buttiglieri et al. ${ }^{16}$ investigated the biomass activity (dosing different organic substrates) calorimetrically, the growth and the decay and pollutants biodegradation in aerobic, anoxic and anaerobic conditions. The use of a high-resolution batch calorimeter (Bio-RC1) made possible to obtain good signal reproducibility under controlled experimental conditions. Moreover it was possible to measure even relatively weak exothermic biological processes, like autotrophic nitrificant or ANAMMOX metabolisms.

Prospects of microcalorimetry in environmental sciences: It is a main content to study the metabolism of living organism in the environmental system for research in environmental sciences. Microcalorimetry has been thought of as potentially useful instrument to continuous monitor the activity of a living process in situ for a long time without disturbing the system. Most of the previous studies using the microcalorimetry method in the environmental science mainly focused on microbial activities and the interactions with organic components and microorganisms. Determination of the heat values of substrate and the living process in a special system with the microcalorimetry method, it is supposed that the microcalorimetry method would facilitate further understanding of the energy balance of the system.

In a special environmental system, the heat release of a living process was affected by many factors. It is felt that a combination of microcalorimeter with different sensors for the measurement of $\mathrm{pH}$ and spectral changes in reaction vessel can be useful in many research applications. It is difficult to incorporate specic analytical sensors into multi-channel microcalorimeters. However, different analytical sensors can be easily incorporated into the flow calorimeters. The modified microcalorimeter with different sensors may provide more valuable information. The study of the combination of material metabolism and energy metabolism in the environmental system can be achieved by detection the special products of microbial metabolism.

\section{ACKNOWLEDGEMENTS}

This work was supported by the National Natural Science Foundation of China (No. 51178484).

\section{REFERENCES}

1. I. Wadsö, Thermochim. Acta, 394, 305 (2002).

2. X.M. Rong, Q.Y. Huang, D.H. Jiang, P. Cai and W. Liang, Pedosphere, 17, 137 (2007).

3. D. Zheng, Y. Liu, Y. Zhang, X.J. Chen and Y.F. Shen, Environ. Toxicol. Pharmacol., 22, 121 (2006).

4. D.L. Fan, X.H. Xiao and X.J. Ma, Thermochim. Acta, 480, 49 (2008)

5. S.X. Zheng, J. Yao, B. Zhao and Z.N. Yu, Eur. J. Soil Biol., 43, 151 (2007).
6. W.-J. Kong, J.-B. Wang, Q.-C. Zang, C. Jin, Z.-W. Wang, X.-Y. Xing, Y.-Y. Wu, Y.-L. Zhao, M.-H. Yang and X.-H. Xiao, J. Chromatogr. B, 879, 3565 (2011).

7. M. Clauss, A. Trampuz, O. Borens, M. Bohner and T. Ilchmann, Acta Biomater, 6, 3791 (2010).

8. T. Selzer, M. Radau and J. Kreuter, Int. J. Pharm., 184, 199 (1999).

9. I. Wadsö, Thermochim. Acta, 294, 1 (1997).

10. Z. Ruming, L. Yi, Z. Zhixiong, S. Ping and Q. Songsheng, J. Biochem. Biophys. Methods, 46, 1 (2000).

11. Y. Liu, X. Li, S.S. Qu and S. Ping, J. Biochem. Biophys. Methods, 45, 231 (2000)

12. H.-Y. Chen, J. Yao, Y. Zhou, H.-L. Chen, F. Wang, N. Gai, R.-Sheng Zhuang, B. Ceccanti, T. Maskow and G. Zaray, J. Hazard. Mater., 159, 465 (2008).

13. J. Yao, L. Tian, Y.X. Wang, A. Djah, F. Wang, H.L. Chen, C.L. Su, R.S. Zhuang, Y. Zhou, M.M.F. Choi and E. Bramanti, Ecotox. Environ. Safety, 69, 289 (2008).

14. F. Aulenta, C. Bassani, J. Ligthart, M. Majone and A. Tilche, Water Res., 36, 1297 (2002).

15. I. Marison, J.-S. Liu, S. Ampuero, U. Von Stockar and B. Schenker, Thermochim. Acta, 309, 157 (1998).

16. G. Buttiglieri, H. Bouju, F. Malpei, E. Ficara and R. Canziani, Biochem. Eng. J., 52, 25 (2010).

17. G. Buttiglieri, F.P. Malpe, E. Daverio, M. Melchiori, H. Nieman and J. Ligthart, Desalination, 178, 211 (2005).

18. E. Daverio, F. Aulenta, J. Ligthart, C. Bassani and A. Rozzi, Water Res., 37, 2723 (2003).

19. N. Barros, I. Gomez-Orellana, S. Feijóo and R. Balsa, Thermochim. Acta, 249, 161 (1995).

20. A.G.S. Prado and C. Airoldi, Thermochim. Acta, 332, 71 (1999).

21. S.A.M. Critter, S.S. Freitas and C. Airoldi, Thermochim. Acta, 417, 275 (2004).

22. N. Barros, S. Fetjoo and R. Balsa, Thermochim. Acta, 296, 53 (1997).

23. S.A.M. Critter, S.S. Freitas and C. Airoldi, Thermochim. Acta, 394, 145 (2002).

24. S.A.M. Critter, S.S. Freitas and C. Airoldi, Thermochim. Acta, 394, 133 (2002)

25. L.N. Regueira, O.N. Fernández, J.A.R. Añón and J.P. Castiñeiras, Thermochim. Acta, 394, 123 (2002).

26. A. Beaubien and C. Jolicoeur, Water Pollut. Control Fed., 57, 95 (1985).

27. C. Jolicoeur, T.C. To, A. Beaubien and R. Samson, Anal. Chim. Acta, 213, 165 (1988).

28. A. Menert, V. Paalme, J. Juhkam and R. Vilu, Thermochim. Acta, 420, 89 (2004).

29. A.G.S. Prado and C. Airoldi, Thermochim. Acta, 371, 169 (2001).

30. A.G.S. Prado and C. Airoldi, Anal. Bioanal. Chem., 376, 686 (2003).

31. F. Wang, J. Yao, H.L. Chen, K. Chen, P. Trebše and G. Zaray, Chemosphere, 78, 319 (2010).

32. R.S. Zhuang, H.L. Chen, J. Yao, Z. Li, J.E. Burnet and M.M.F. Choi, J. Hazard. Mater, 189, 323 (2011).

33. H.L. Chen, J. Yao, F. Wang, M.M.F. Choi, E. Bramanti and G. Zaray, J. Hazard. Mater, 167, 846 (2009).

34. H. Guo, J. Yao, M.M. Cai, Y.G. Qian, Y. Guo, H.H. Richnow, R.E. Blake, S. Doni and B. Ceccanti, Chemosphere, 87, 1273 (2012).

35. S.M. McGuinness and B.G. Barisas, Environ. Sci. Technol., 25, 1092 (1991).

36. F. Wang, J. Yao, H.L. Chen, Y. Zhou, Y.J. Chen, H.Y. Chen, N. Gai, R.S. Zhuang, L. Tian, T. Maskow, B. Ceccanti, P. Trebse and G. Zaray, Ecotox. Environ. Safety, 72, 128 (2009).

37. Y. Zhou, J. Yao, M.M.F. Choi, Y.J. Chen, H.Y. Chen, R. Mohammad, R.S. Zhuang, H.L. Chen, F. Wang, T. Maskow and G. Zaray, J. Hazard. Mater, 875, 169 (2009).

38. F. Wang, J. Yao, Y. Si, H.L. Chen, M. Russel, K. Chen, Y.G. Qian, G. Zaray and E. Bramanti, J. Hazard. Mater, 173, 510 (2010).

39. E. Daverio, H. Spanjers, C. Bassani, J. Ligthart and H. Nieman, Biotechnol. Bioeng., 82, 499 (2003).

40. H.L. Chen, J. Yao, F. Wang, Y. Zhou, K. Chen, R.S. Zhuang, M.M.F. Choi and G. Zaray, Sci. Total. Environ., 408, 1043 (2010). 